\title{
Can Automated Road Vehicles Harmonize with Traffic Flow While Guaranteeing A Safe Distance?
}

\author{
Matthias Althoff and Robert Lösch
}

\begin{abstract}
A frequently raised argument against safelydriving automated vehicles is that they would not harmonize well with traffic flow-unrealistically large headways would invite other traffic participants to cut in and thus put passengers of following automated vehicles at risk. In order to test this hypothesis, we use real data of thousands of vehicles recorded in the United States as part of the Next Generation Simulation (NGSIM) program. To study the hypothesis, we pretend each human-driven vehicle is automated: These automated vehicles drive exactly as the recorded human drivers, but they have a much smaller reaction time and thus can still drive safely in situations that are unsafe for human drivers. The main result is that in only very few cases an automated vehicle would not drive safely, although more than half of the human drivers do not keep a safe distance according to their capabilities. Thus, there is no increased risk of vehicles cutting in-and if they do, automated vehicles are only at risk in around $10 \%$ of all cases compared to around $60 \%$ for human drivers.
\end{abstract}

\section{INTRODUCTION}

In recent years, a significant effort has been made to ensure that intelligent road vehicles become safer by developing novel collision avoidance systems (see e.g. [1]). It is often criticized that automated vehicles adhering to a safe distance do not harmonize with traffic flow. The most prominent example is that on a highway, automated vehicles would keep an unrealistically large headway to ensure collision avoidance when the preceding vehicle fully brakes. This could invite many other traffic participants to merge in front of the considered automated vehicle (ego vehicle) and thus temporarily put it at risk since the headway is not safe anymore as depicted in Fig. 1 This hypothesis is motivated by studies showing that many human drivers often do not keep a sufficient distance [2], [3]. In [4] it is presented that the willingness to drive with unsafe headway increases with traffic density. Thus, some people advocate to intentionally keep an unsafe distance, since this is supposed to be safer compared to vehicles merging in front of the ego vehicle to prevent the situation in Fig. 10 To the best knowledge of the authors, no previous study has investigated this question. We consider data of movements of traffic participants on a highway and a boulevard. Since automated vehicles have a much smaller reaction delay than human drivers, automated vehicles can drive closer without compromising on safety. The question is whether this distance is short enough to harmonize with current traffic flow.

Matthias Althoff and Robert Lösch are with the Technische Universität München, Fakultät für Informatik, Lehrstuhl für Robotik und Echtzeitsysteme, Boltzmannstraße 3, 85748, Garching, Germany. \{althoff, robert.loesch\}etum. de
There exists a lot of work on how mixed human/automated traffic affects traffic flow [5]. However, no work considers the effects of merging vehicles on safety when a safe gap is kept. In [6] the authors conclude that based on computer simulations, automated vehicles have a positive effect on traffic flow. It has further been shown that even a small penetration of automated vehicles can already achieve such a positive effect [7]. However, when the accelerations of automated vehicles are restricted to the ones of rail systems for comparison of comfort, the throughput at intersections decreases [8]. A control strategy to ensure safe driving when combining adaptive cruise control (ACC) with a safety controller is described in [9]. The effects of cut-ins due to the use of ACC on traffic flow are investigated in [10]. This study, however does not consider the effects on safety (i.e. what is the percentage of cases in which the vehicle is at risk) and does not use a large set of real traffic data, but simulations and field experiments. Different spacing policies have been evaluated in [11], but the effects of cut-ins are not considered. Limitations of computer simulations compared to real traffic data is discussed in [12]. Recent work on modeling human behavior tries to close the reality gap by utilizing machine learning techniques; see e.g. [13], [14]. An average model for various control concepts of automated vehicles is proposed in [15].

Our contribution is a first study showing how well a safelydriving automated vehicle harmonizes with traffic flow based on data from several thousand recorded traffic participants. In particular, we provide insight into when other traffic participants merge and whether those merges put other vehicles at risk.

\section{OVERVIEW OF THE APPROACH}

The basis of our investigation is the computation of safe distances. The Vienna Convention on Road Traffic [16], which has been ratified by most countries, defines in article $13 \S 5$ safe distance as: "The driver of a vehicle moving behind another vehicle shall keep at a sufficient distance from that other vehicle to avoid collision if the vehicle in front should suddenly slow down or stop." Based on this informal definition, we compute the safe distance in Sec. IIII

The safe distance is compared to distances between vehicles observed in real traffic situations in Sec. IV] We use the recorded vehicle data from the Next Generation Simulation (NGSIM) program [17] to generate histograms of the distance between traffic participants in relation to their safe distance. In particular, we consider that automated vehicles have a smaller reaction time leading to a shorter safe distance, which 


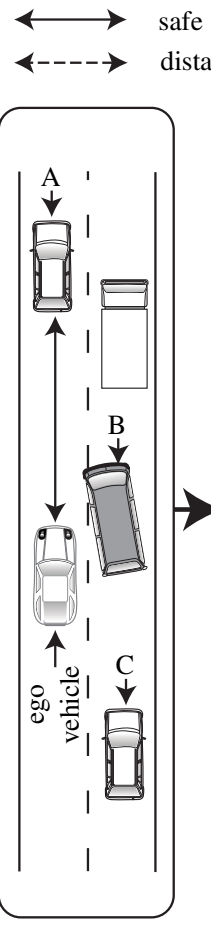

time $t_{1}$

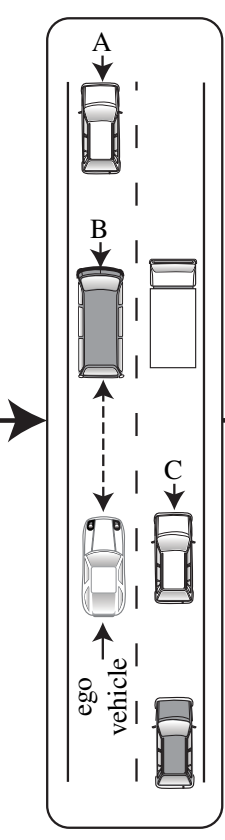

time $t_{2}$

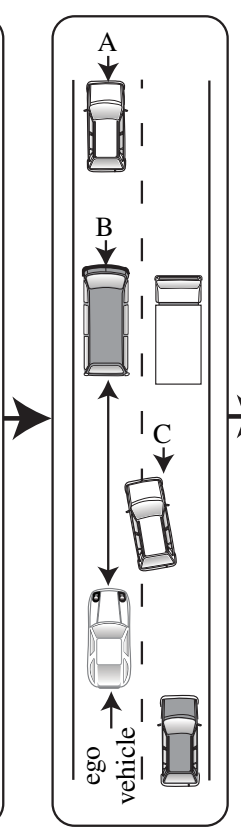

time $t_{3}$

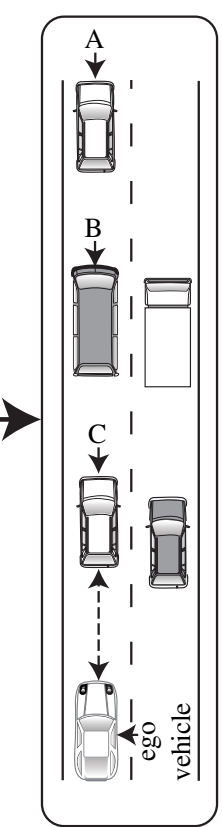

time $t_{4}$
Fig. 1. Danger of cutting-in vehicles: A safe distance towards preceding traffic participants might invite others to cut in, causing dangerously low distances after merging. This hypothesis is illustrated by several snapshots. Time $t_{1}$ : The ego vehicle keeps a safe distance to the preceding vehicle A. Time $t_{2}$ : Vehicle $\mathrm{B}$ has merged due to the relatively large distance between the ego vehicle and vehicle $\mathrm{A}$ in comparison to distances between surrounding vehicles. This results in a dangerously low distance between the ego vehicle and vehicle B. Time $t_{3}$ : The ego vehicle further slows down until the distance is safe again. Time $t_{4}$ : The next vehicle (vehicle $\mathrm{C}$ ) has merged due to the safe distance between the ego vehicle and vehicle $B$. This might be a reoccurring pattern.

we refer to as machine safe distance. The safe distance for humans is referred to as human safe distance and the actual average distance is referred to as human average distance. If the machine safe distance is similar to the human average distance, we can assume that automated vehicles harmonize well with human drivers. The focus on reaction time is justified by many studies e.g. [18], [19] that show that reaction time is the main factor when considering traffic on highways and boulevards as done in this work. Smoother velocity profiles as expected for automated vehicles are not considered a major safety benefit. Finally, we analyze the conditions under which other traffic participants merge and whether the now shorter gap to a following automated vehicle would put it at risk, i.e., if the new gap is below the machine safe distance.

\section{SAFE Distance}

Since our data is obtained from a highway and a boulevard with negligible inclination and curvature (see Sec. IV-A], it suffices to consider the simplified longitudinal dynamics $\ddot{s}=a$, where $s$ is the position and $a$ is the longitudinal acceleration, both in driving direction [11]. Since the safe distance is computed based on the deceleration potential, we assume that vehicles will apply maximum negative acceleration once the braking process has been initiated. Negative acceleration is limited by the maximum tire-road friction because the brake system is dimensioned so that maximum tire forces can be utilized. Since tire-road friction only marginally varies between different compounds and makers for the same road and weather condition [20, Tab. 4], [21, Fig. 3.2], we assume an acceleration potential of $a_{\max }$ for a given road and weather condition. In our study, the road and weather conditions are constant so that we can assume the constant acceleration potential $a_{\max }$ for all detected vehicles.

Without loss of generality, we assume that the preceding vehicle fully brakes at time zero, while the following vehicle continues with constant velocity before braking after a reaction delay $\delta$. Since we assume constant accelerations over time, we can utilize the well-known solution for constant acceleration $s(t)=s_{0}+v_{0} t+\frac{1}{2} a t^{2}$, where $s_{0}$ is the initial position, $v_{0}$ is the initial velocity, and $t$ is time. We further introduce the time $t_{p, s t o p}$ and $t_{f, s t o p}$ when the preceding and following vehicle stop, respectively, and we define $t_{\text {stop }}=\max \left(t_{p, \text { stop }}, t_{f, \text { stop }}\right)$. Both vehicles do not collide if

$$
\begin{aligned}
& \forall t \in\left[0, t_{\text {stop }}\right]: \quad s_{p}(t)-s_{f}(t) \geq 0 \\
& s_{p}(t)= \begin{cases}s_{p}\left(t_{p, s t o p}\right), & t \in\left[t_{p, \text { stop }}, t_{\text {stop }}\right] \\
s_{p, 0}+v_{p, 0} t-\frac{1}{2} a_{\text {max }} t^{2}, t \in\left[0, t_{p, \text { stop }}\right]\end{cases} \\
& s_{f}(t)= \begin{cases}s_{f}\left(t_{f, \text { stop }}\right), & t \in\left[t_{f, \text { stop }}, t_{\text {stop }}\right] \\
s_{f, 0}+v_{f, 0} t-\frac{1}{2} a_{\text {max }}(t-\delta)^{2}, & t \in\left[\delta, t_{f, \text { stop }}\right] \\
s_{f, 0}+v_{f, 0} t, & t \in[0, \delta] .\end{cases}
\end{aligned}
$$

Although previous work on safe vehicle distances exists (see e.g. [22], [23]), we have not found a provably correct safe distance calculation considering time delay.

We first derive the solution for the case that both vehicles can have different acceleration $a_{p}$ and $a_{f}$ and later discuss in Prop. 1 the special case that $a_{p}=a_{f}$. It is possible that both vehicles touch each other (i.e. are about to crash) at a time $t_{\text {touch }} \leq t_{p, \text { stop }}$. The closed-form solution of $t_{\text {touch }}$ for $t_{\text {touch }}>\delta$ follows from demanding $s_{p}\left(t_{\text {touch }}\right)=s_{f}\left(t_{\text {touch }}\right)$ and inserting $s_{p}\left(t_{\text {touch }}\right)=s_{p, 0}+v_{p, 0} t_{\text {touch }}+\frac{1}{2} a_{p} t_{\text {touch }}^{2}$, $s_{f}\left(t_{\text {touch }}\right)=s_{f, 0}+v_{f, 0} t_{\text {touch }}+\frac{1}{2} a_{f}\left(t_{\text {touch }}-\delta\right)^{2}$ :

$$
\begin{aligned}
& \underbrace{s_{p, 0}-s_{f, 0}-\frac{1}{2} a_{f} \delta^{2}}_{=: \Delta \tilde{s}_{0}}+(\underbrace{v_{p, 0}-v_{f, 0}+a_{f} \delta}_{=: \Delta \tilde{v}_{0}}) t_{\text {touch }} \\
& +\frac{1}{2}(\underbrace{a_{p}-a_{f}}_{=: \Delta a}) t_{\text {touch }}^{2}=0 \\
& \Rightarrow t_{\text {touch }}= \begin{cases}\frac{-\Delta \tilde{v}_{0} \pm \sqrt{\Delta \tilde{v}_{0}^{2}-2 \Delta a \Delta \tilde{s}_{0}}}{\Delta a}, & \text { for } \Delta a \neq 0, \\
\frac{-\Delta \tilde{s}_{0}}{\Delta \tilde{v}_{0}}, & \text { for } \Delta a=0 .\end{cases}
\end{aligned}
$$

For the computation of $t_{\text {touch }}$, it is assumed that vehicles accelerate backwards once they have stopped. Thus, for $t_{\text {touch }} \geq t_{p, \text { stop }}$, we instead have to consider the positions 
when the vehicles have stopped. In total, there are four cases, where $\wedge$ represents the logical and:

1) $0 \leq t_{\text {touch }}<t_{p, \text { stop }}$ : A crash occurs while both vehicles are moving.

2) $\left(t_{\text {touch }} \geq t_{p, \text { stop }}\right) \wedge\left(s_{p}\left(t_{p, \text { stop }}\right)<s_{f}\left(t_{f, \text { stop }}\right)\right)$ : The following vehicle crashes with the stopped preceding vehicle.

3) $\left(t_{\text {touch }} \geq t_{p, \text { stop }}\right) \wedge\left(s_{p}\left(t_{p, \text { stop }}\right) \geq s_{f}\left(t_{f, \text { stop }}\right)\right)$ : No crash occurs.

4) $t_{\text {touch }}<0$ : No crash occurs.

Due to constant acceleration, the stopping times are

$$
t_{p, \text { stop }}=\frac{v_{p, 0}}{a_{\max }}, \quad t_{f, \text { stop }}=\frac{v_{f, 0}}{a_{\max }}+\delta .
$$

Inserting (3) into $s_{p}\left(t_{p, s t o p}\right)=s_{f}\left(t_{f, s t o p}\right)$ and using (1) results after simple rewriting in a required, minimum initial distance $\Delta s_{\min , 0}$, which is obtained as

$$
\Delta s_{\min , 0}=\frac{1}{2 a_{\max }}\left(v_{f, 0}^{2}-v_{p, 0}^{2}\right)+v_{f, 0} \delta .
$$

To ensure that $\Delta s_{\min , 0} \geq 0$, we require the condition that

$$
v_{p, 0} \leq \sqrt{v_{f, 0}^{2}+2 v_{f, 0} \delta a_{\max }},
$$

which is trivially obtained from (4). We can now formulate the following simplification:

\section{Proposition 1 (Final position check is sufficient):}

When both vehicles use the same negative acceleration $a_{p}=a_{f}=-a_{\max }$ and (5) holds, it suffices to check whether $s_{p}\left(t_{p, s t o p}\right)=s_{f}\left(t_{f, s t o p}\right)$ without additionally checking whether $t_{\text {touch }} \geq t_{p \text {,stop }}$ to obtain the safe distance, which we formally write as

Eq. (5) $\wedge\left(s_{p}\left(t_{p, s t o p}\right)=s_{f}\left(t_{f, \text { stop }}\right) \Rightarrow\left(t_{\text {touch }} \geq t_{p, \text { stop }}\right)\right.$.

Proof: Inserting (2) for $a_{p}=a_{f}=-a_{\max }$ and (3) into $t_{\text {touch }} \geq t_{p, \text { stop }}$ results in

$$
\frac{-\left(\Delta s_{\min , 0}+\frac{1}{2} a_{\max } \delta^{2}\right)}{v_{p, 0}-v_{f, 0}-a_{\max } \delta} \geq \frac{v_{p, 0}}{a_{\max }} .
$$

Inserting (4) yields

$$
\frac{-\left(\frac{1}{2 a_{\max }}\left(v_{f, 0}^{2}-v_{p, 0}^{2}\right)+v_{f, 0} \delta+\frac{1}{2} a_{\max } \delta^{2}\right)}{v_{p, 0}-v_{f, 0}-a_{\max } \delta} \geq \frac{v_{p, 0}}{a_{\max }} .
$$

After finding the root for $v_{p, 0}$ of the corresponding equality of (6), we can state that the inequality (6) is fulfilled for

$$
v_{p, 0} \leq v_{f, 0}+\delta a_{\max } .
$$

Next, using (5) we show that

$$
\begin{aligned}
v_{p, 0} & \leq \sqrt{v_{f, 0}^{2}+2 v_{f, 0} \delta a_{\max }} \\
& =\sqrt{\left(v_{f, 0}+\delta a_{\max }\right)^{2}-\left(\delta a_{\max }\right)^{2}} \leq v_{f, 0}+\delta a_{\max } .
\end{aligned}
$$

From (8) we can conclude that (7) is always fulfilled and thus, $t_{\text {touch }} \geq t_{p, \text { stop }}$ always holds.

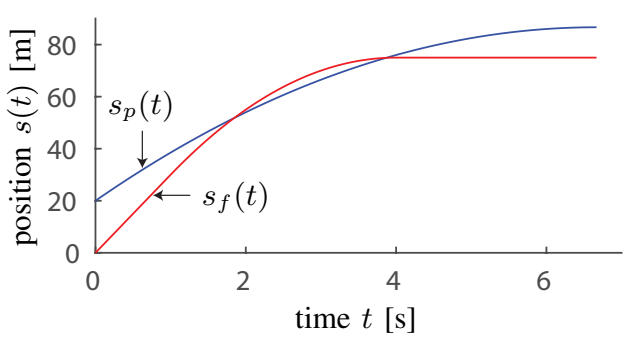

Fig. 2. When $a_{p} \neq a_{f}$, it does not follow that $t_{t o u c h} \geq t_{p \text {,stop }}$ for $s_{p}\left(t_{p, s t o p}\right)>s_{f}\left(t_{f, s t o p}\right)$; parameters: $s_{p, 0}=20 \mathrm{~m}, v_{p, 0}=20 \mathrm{~m} / \mathrm{s}$, $a_{p}=-3 \mathrm{~m} / \mathrm{s}^{2}, s_{f, 0}=0 \mathrm{~m}, v_{f, 0}=30 \mathrm{~m} / \mathrm{s}, a_{f}=-10 \mathrm{~m} / \mathrm{s}^{2}, \delta=1 \mathrm{~s}$.

Since we know from Prop. 1 that it is sufficient to check $s_{p}\left(t_{p, s t o p}\right)=s_{f}\left(t_{f, s t o p}\right)$, it follows from (4) that

$$
s_{\text {safe }}\left(v_{p, 0}, v_{f, 0}, a_{\max }, \delta\right)=\frac{1}{2 a_{\max }}\left(v_{f, 0}^{2}-v_{p, 0}^{2}\right)+v_{f, 0} \delta \text {. }
$$

Please note that (5) is automatically fulfilled for $s_{\text {safe }}\left(v_{p, 0}, v_{f, 0}, a_{\max }, \delta\right) \geq 0$ and that for $a_{p} \neq a_{f}$, Prop. 1 does not hold as shown in Fig. 2] The safe distance is used in the next section to analyze how well a safely-driving automated vehicle would harmonize with traffic flow.

\section{Evaluation of Real Traffic Data}

We now use the safe distance $s_{\text {safe }}\left(v_{p, 0}, v_{f, 0}, a_{\max }, \delta\right)$ from (9) to compare it with measured distances between traffic participants in real world scenarios. In Sec. IV-A we introduce the used traffic data. Assumptions and the parameter selection are justified in Sec. IV-B To what extend other traffic participants keep a safe distance is evaluated in Sec. IV-C] Finally, in Sec IV-D we particularly analyze merging vehicles and whether the new distance of a following automated vehicle provides a threat.

\section{A. Traffic Data}

As previously mentioned, we use the data from the Next Generation Simulation (NGSIM) program to check how well a safely-driving autonomous vehicle would harmonize with traffic flow. The data has originally been collected to validate microscopic traffic simulations, i.e. traffic simulations where individual vehicles are considered as opposed to abstract traffic flows. Vehicles have been detected by several digital video cameras along distinctive road sections in the United States as illustrated in Fig. 3. We consider the following datasets:

- Lankershim Boulevard Dataset: The detected area consists of three to four lanes in each direction on a boulevard and a complete coverage of three signalized intersections. The area is around 500 meters in length and 2442 vehicles have been recorded on June 16, 2005 from 8:30 am to 9:00 am.

- US Highway 101 Dataset: The study area is approximately 640 meters long and consists of five mainline lanes on a highway including an on-ramp and an offramp. The dataset contains 6101 vehicles which have 
been recorded on June 15, 2005 from 7:50 am to 8:35 am.

Of all sections, the original vehicle data as well as the video images with tracked vehicles are available [17]. The data of each detected vehicle is updated every 0.1 seconds for which we list the most relevant data items: position ( $\mathrm{x}$ - and $\mathrm{y}-$ coordinates), velocity, acceleration, occupied lane, preceding vehicle ID, following vehicle ID, spacing to the preceding vehicle, and time headway to the preceding vehicle. For each vehicle, we also have the vehicle dimensions (length and width) and the vehicle class (motorcycle, car, or truck).

\section{B. Model Assumptions}

As already discussed in Sec. III, tire-road friction only varies marginally between different compounds and makers [20, Tab. 4], [21, Fig. 3.2], but is heavily influenced by road and weather conditions. These, however, are fairly homogeneous throughout the observation areas described in Sec. IV-A Since the video data shows dry surface on a good road, we have chosen a friction coefficient of $\mu=0.82$, which corresponds to $a_{\max }=8 \frac{\mathrm{m}}{\mathrm{s}^{2}}$ when assuming a gravity constant of $9.8 \mathrm{~m} / \mathrm{s}^{s}$ [21, Fig. 3.3].

We further have to choose a proper reaction time for humans $\delta_{\text {human }}$ and for automated vehicles $\delta_{\text {machine }}$. Ultimately, we are interested in studying how safe people would be in an automated vehicle that would drive like humans, except that we assume the reaction $\delta_{\text {machine. }}$ Consequently, the reaction delay of humans is only used for comparison to see the impact of varying time delay and thus does not need to be known exactly. Studies indicate that most people have a reaction time of around $1 \mathrm{~s}$, see [24, Fig. 3], [25, Fig. 1], [26, Fig. 1]. The time required to apply full braking is longer since human drivers are often braking initially too carefully in emergency situations. In [27] the authors obtain an average reaction time to full braking of $2.2 \mathrm{~s}$ in a driver simulator and $2.3 \mathrm{~s}$ on a test track. Since drivers already brake before the full brake pressure is applied, we choose $\delta_{\text {human }}=2 \mathrm{~s}$ as a compromise. In [28] it is investigated how the reaction time varies when adaptive cruise control is used. In that work, it is also shown that the results of many studies with respect to reaction time vary.

Contrary to human reaction time, there is no work that investigates the average reaction time of automated vehicles, to the best of our knowledge. This is probably because no automated vehicle capable of performing emergency braking at higher speeds is commercially available and thus no market study has been performed. We will assume $\delta_{\text {machine }}=0.3 \mathrm{~s}$ from previous experience of the authors with autonomous vehicles, see e.g. [29], [30]. It is later shown in Tab. II] that the results are similar for $\delta_{\text {machine }}=0.2 \mathrm{~s}$ and $\delta_{\text {machine }}=0.4 \mathrm{~s}$. Since we follow a fundamental discussion about integration of automated vehicles, we further assume that the automated vehicle can track all surrounding vehicles and no significant additional reaction delay is introduced due to the perception deficiencies.

\section{Evaluation of Safe Distance}

In this subsection, we investigate whether human drivers keep a safe distance and how safe the distance would be if an automated vehicle would drive like a human. We denote the recorded distance by $s_{\text {measured }}$ and evaluate the spacing relative to the safe distance obtained from (9):

$$
\Delta s_{r e l}\left(v_{p, 0}, v_{f, 0}, a_{\max }, \delta\right)=\frac{s_{\text {measured }}}{s_{\text {safe }}\left(v_{p, 0}, v_{f, 0}, a_{\max }, \delta\right)} .
$$

The normalization makes it possible to compare the data across different velocities, relative velocities, and spacings between vehicles. To formally describe the considered data set, we introduce the safe distance $\Delta s_{r e l, i, k}$ for the $i^{t h}$ vehicle and the $k^{t h}$ point in time. Let us denote the set of vehicle IDs from 1 to $N$ as $\mathcal{I}$ and the set of time indices $s_{i} \in \mathbb{N}^{+}$to $f_{i} \in \mathbb{N}^{+}$for the $i^{t h}$ vehicle as $\mathcal{K}_{i}=\left\{s_{i}, \ldots, f_{i}\right\}$. The presented histograms show the set of safe distances

$$
\mathcal{S}_{\text {distance }}=\left\{\Delta s_{\text {rel }, i, k} \mid i \in \mathcal{I}, k \in \mathcal{K}_{i}\right\} .
$$

The histograms for $\mathcal{S}_{\text {distance }}$ of the Lankershim Boulevard and the US Highway 101 are shown for $\delta=\delta_{\text {human }}=2 \mathrm{~s}$ and $\delta=\delta_{\text {machine }}=0.3 \mathrm{~s}$ in Fig. 4. It can be clearly seen that most human drivers do not keep a sufficient distance to the preceding vehicle. When the automated vehicles drive exactly as the human-driven ones, but with the much smaller reaction time $\delta_{\text {machine, }}$, there is a dramatic shift in the histogram: Now, in most cases, the automated vehicles would drive safely. Of course, an automated vehicle would never intentionally keep an unsafe distance, but in only a few cases the behavior would have to be slightly adapted to always achieve $\Delta s_{\text {rel }} \geq 1$.

The percentage of vehicles in the range $0 \leq \Delta s_{\text {rel }} \leq 5$ driving in the unsafe range $0 \leq \Delta s_{\text {rel }} \leq 1$ is shown in Tab. I. It can be seen that the percentage of unsafelydriving vehicles drops significantly from $51.63 \%$ to $3.26 \%$ for the Lankershim data and from $61.02 \%$ to $1.09 \%$ for the US Highway 101 data, if the vehicles are automated. Similar results are obtained for varying $\delta_{\text {machine }}$ in Tab. II In conclusion, automated vehicles would harmonize well with current traffic flow, while driving safely. It remains to check in this paper if merging vehicles would pose a risk to autonomous vehicles driving human-like.

TABLE I

PeRCENTAge OF Vehicles WITH $\Delta s_{r e l} \leq 5$ DRIVING UNSAFE

$$
\left(\Delta s_{\text {rel }}<1\right) \text {. }
$$

\begin{tabular}{lcccccccc}
\hline & \multicolumn{2}{c}{ lane following } & & \multicolumn{2}{c}{ before merging } & & \multicolumn{2}{c}{ after merging } \\
\cline { 2 - 3 } & L. Blvd & US 101 & & L. Blvd & US 101 & & L. Blvd & US 101 \\
\hline human & $51.63 \%$ & $61.02 \%$ & & $23.55 \%$ & $28.88 \%$ & & $48.29 \%$ & $78.60 \%$ \\
machine & $3.26 \%$ & $1.09 \%$ & & $0.89 \%$ & $0.00 \%$ & & $10.62 \%$ & $14.12 \%$ \\
\hline
\end{tabular}

\section{Evaluation of Merging}

This subsection investigates the risk distribution when vehicles merge. As for the safe distance evaluation, we use the relative safe distance as introduced in (10). The difference 


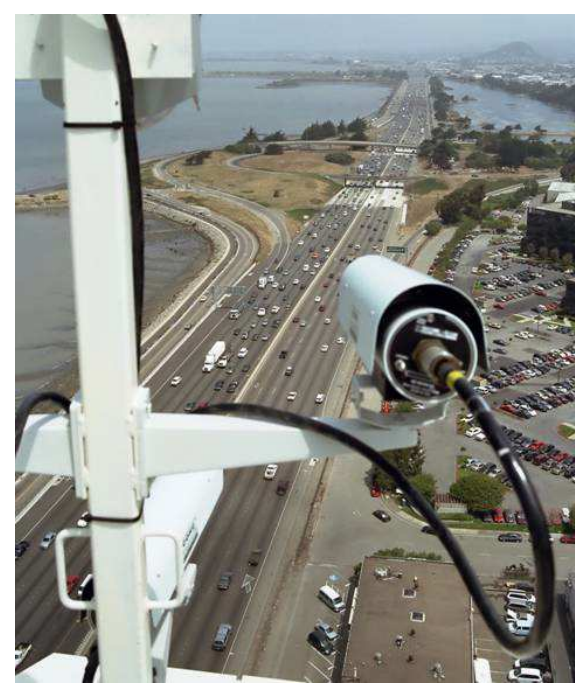

(a) Camera setup taken from [17].

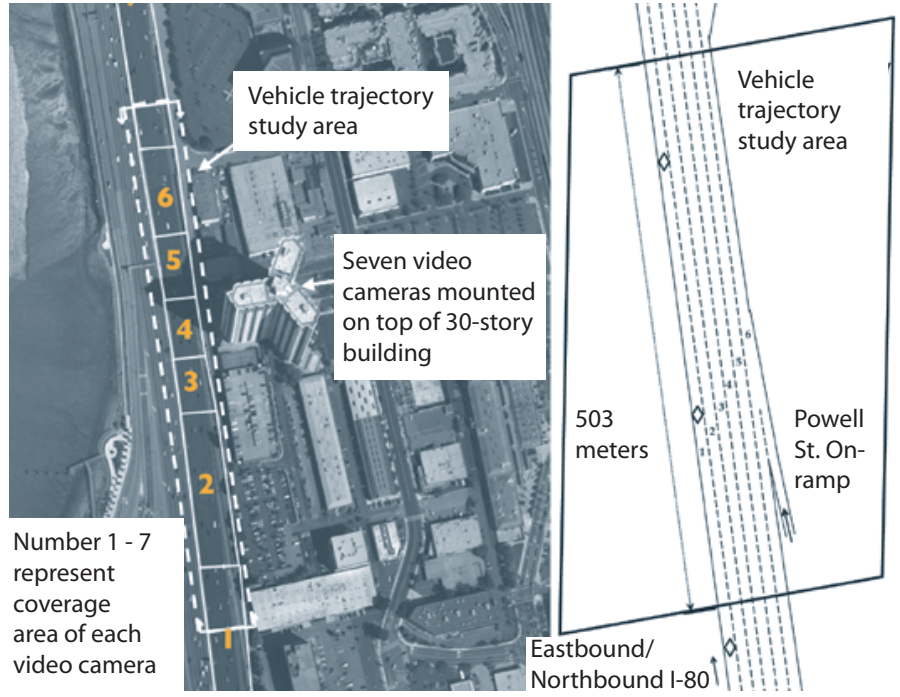

(b) Study area and coverage of individual cameras taken from http://ngsim.fhwa.dot.gov/

Fig. 3. Camera setup and corresponding study area to record individual vehicles.

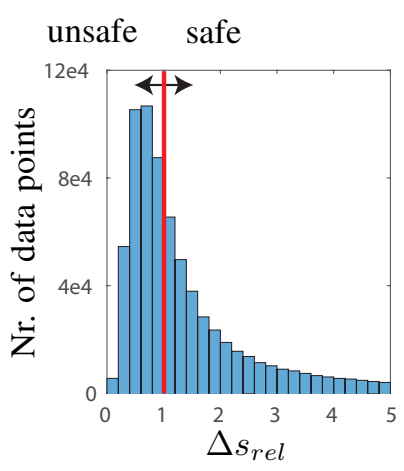

(a) Lankershim Blvd, $\delta=2 \mathrm{~s}$.

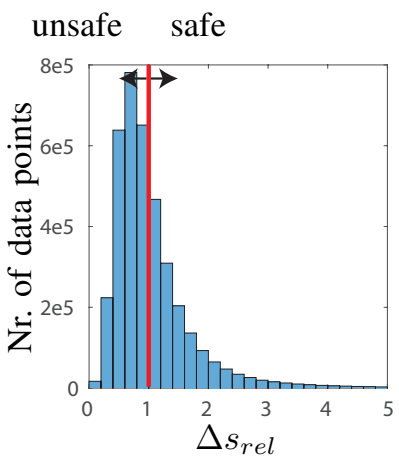

(c) US Highway $101, \delta=2 \mathrm{~s}$.

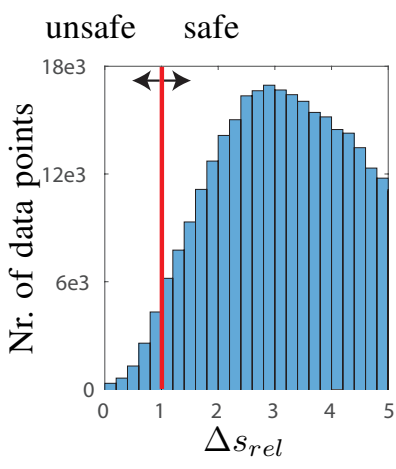

(b) Lankershim Blvd, $\delta=0.3 \mathrm{~s}$.

unsafe safe

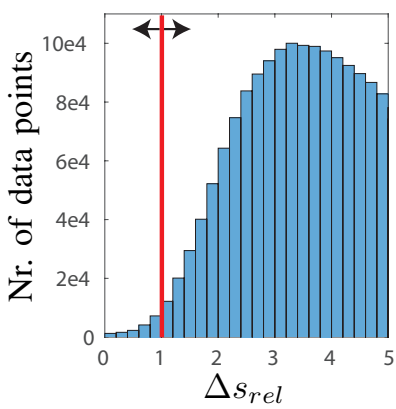

(d) US Highway $101, \delta=0.3 \mathrm{~s}$.

Fig. 4. Histograms of relative safe distance $\Delta s_{r e l}$.

is that we only consider the points in time of a vehicle, when it changes the lane. This point in time is determined by checking whether the vehicle center has crossed the lane divider. All time indices when the $i^{t h}$ vehicle has changed a lane are stored in $\mathcal{K}_{\text {merge }, i}$. The data set to evaluate merging

TABLE II

Percentage of Vehicles WITH $\Delta s_{r e l} \leq 5$ DRIVING UNSAFE $\left(\Delta s_{r e l}<1\right)$ FOR VARYING DELAY TIMES $\delta_{\text {machine }}$.

\begin{tabular}{lccc}
\hline & $0.2 \mathrm{~s}$ & $0.3 \mathrm{~s}$ & $0.4 \mathrm{~s}$ \\
\hline L. Blvd & $2.86 \%$ & $3.26 \%$ & $4.12 \%$ \\
US 101 & $1.09 \%$ & $1.09 \%$ & $1.46 \%$
\end{tabular}

is modified from 11 to

$$
\mathcal{S}_{\text {merge }}=\left\{\Delta s_{\text {rel }, i, k} \mid i \in \mathcal{I}, k \in \mathcal{K}_{\text {merge }, i}\right\} .
$$

We first investigate the gap of a vehicle to its preceding vehicle just before another vehicle merges. This would be the distance between vehicle $\mathrm{A}$ and the ego vehicle at some time $t^{*} \in\left[t_{1}, t_{2}\right]$ in Fig. 1, where $t^{*}$ is exactly when the center of vehicle $\mathrm{B}$ crosses the lane divider. We also consider the gap at $t^{*}$ between the newly preceding vehicle and the following vehicle, which is the one between vehicle $\mathrm{B}$ and the ego vehicle in Fig. 1

The histogram in Fig. 5 for the distances just before a vehicle merges shows that a fair number of traffic participants even move into a gap, although the existing gap is already unsafe. This is quite astonishing and shows that it will be almost impossible for automated vehicles to avoid vehicles merging in an unsafe manner. Such a behavior requires that the automated vehicle immediately increases the gap, while the vehicle merges.

The distribution of the new gaps just after merging are presented in Fig. 6. The results show that merging vehicles often put following vehicles at risk. Compared to the distances during normal driving shown in Fig. 4, the probability has increased for very short distances: The percentage of vehicles with $\Delta s_{\text {rel }} \leq 5$ keeping an extremely low relative distance of $\Delta s_{r e l}<0.5$ has increased from $15.85 \%$ to $23.88 \%$ for 


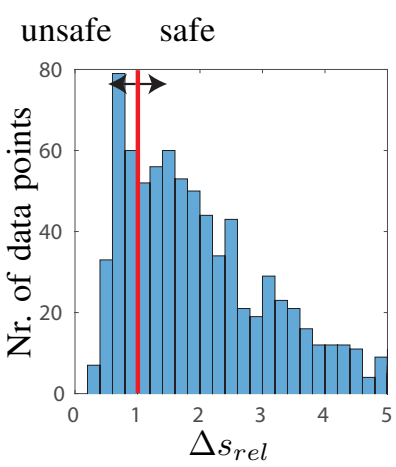

(a) Lankershim Blvd, $\delta=2 \mathrm{~s}$.

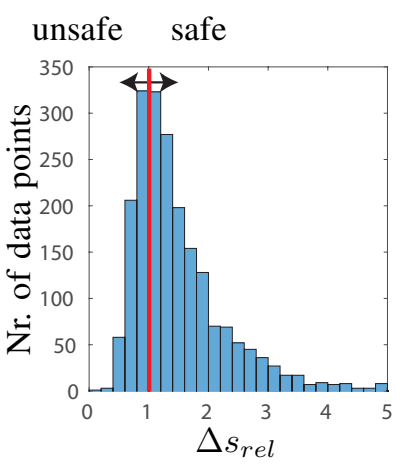

(c) US Highway $101, \delta=2 \mathrm{~s}$.

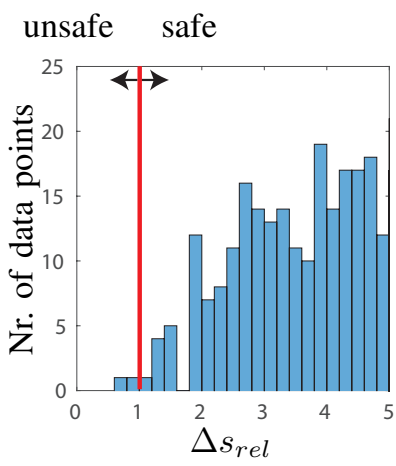

(b) Lankershim Blvd, $\delta=0.3 \mathrm{~s}$.

unsafe safe

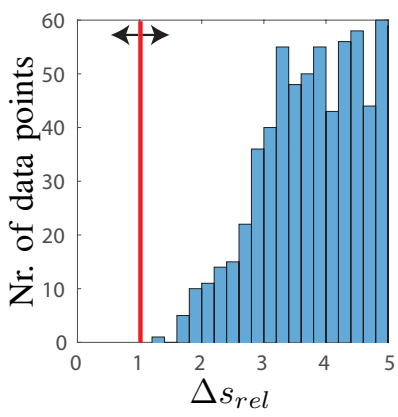

(d) US Highway $101, \delta=0.3 \mathrm{~s}$.

Fig. 5. Histograms of safe distance just before a vehicle merges.

the Lankershim Boulevard and drastically from $13.66 \%$ to $42.17 \%$ for the US Highway 101 . The distance after merging would be much less of a problem when a vehicle merges in front of an automated vehicle: Due to the shorter reaction time of automated vehicles, there is a broad distribution for $s_{\text {rel }} \geq 1$, while far fewer vehicles would be at risk as shown in Tab. [ Assuming automation, the percentage of unsafely-driving vehicles, after a vehicle merged in front, improved from $48.29 \%$ to $10.62 \%$ for the Lankershim data and from $78.60 \%$ to $14.12 \%$ for the US Highway 101 data. Interestingly, the numbers do not drop as much as in the case when vehicles just follow a lane, although the percentage of unsafely-driving humans after a merging situation differs only a little from lane following. The reason is, as previously discussed, that the percentage of very unsafe vehicles with $\Delta s_{\text {rel }}<0.5$ has drastically increased.

\section{CONCLUSION}

This paper presents a first study on how safely-automated vehicles would drive if they kept the same distances to preceding vehicles as human drivers. Although human drivers following vehicles within five times the safe distance are not keeping a safe distance in more than $50 \%$ of all cases, an automated vehicle would only drive risky in a few cases (3\% on Lankershim Blvd and 1\% on US Highway 101). Of course, those numbers are varying between different traffic settings and countries, but the significant safety gain shows that automated vehicles keeping a safe distance can well harmonize with existing traffic due to reduced reaction time. When vehicles are merging, they typically put the following

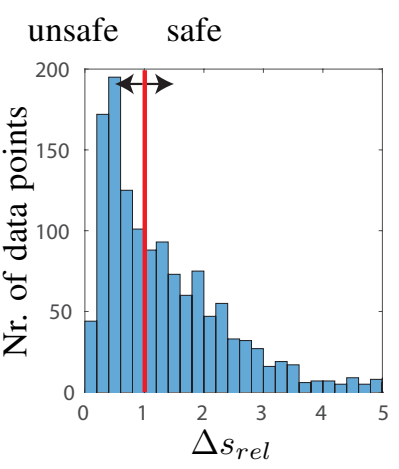

(a) Lankershim Blvd, $\delta=2 \mathrm{~s}$.

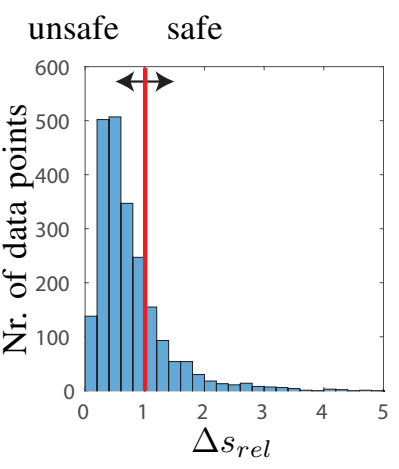

(c) US Highway $101, \delta=2 \mathrm{~s}$.

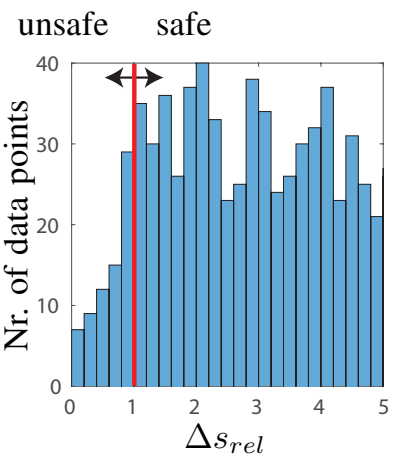

(b) Lankershim Blvd, $\delta=0.3 \mathrm{~s}$.

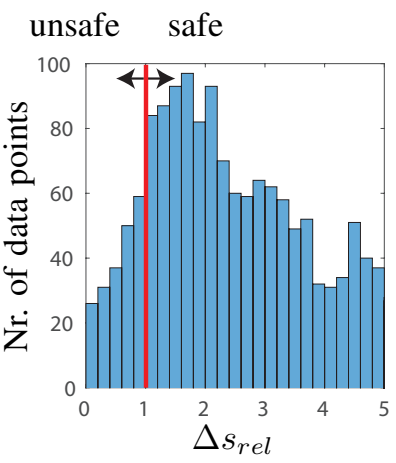

(d) US Highway $101, \delta=0.3 \mathrm{~s}$.
Fig. 6. Histograms of safe distance just after a vehicle has merged.

vehicle at risk. In our data, unsafe situations reduced for automated vehicles from $48.29 \%$ to $10.62 \%$ for Lankershim Blvd and from $78.60 \%$ to $14.12 \%$ for US Highway 101.

Thus, we can conclude that automated vehicles driving safely harmonize well with existing traffic. It is impossible for an automated vehicle to eliminate all risk when a vehicle cuts in. However, since automated vehicles could immediately slow down when detecting an initiated merge, the numbers could possibly be reduced to only a few cases, depending on how much deceleration is accepted by the passengers for comfort reasons. It would be unreasonable to perform full braking if a vehicle would recklessly cut in just to immediately restore a safe distance.

\section{ACKNOWLEDGEMENTS}

We gratefully acknowledge partial financial support by the German Research Foundation (DFG) projects Graduiertenkolleg 1480 (PUMA) and AL 1185/3-1.

\section{REFERENCES}

[1] K. D. Kusano and H. C. Gabler, "Safety benefits of forward collision warning, brake assist, and autonomous braking systems in rear-end collisions," IEEE Transactions on Intelligent Transportation Systems, vol. 13, no. 4, pp. 1546-1555, 2012.

[2] J.-H. Wang and M. Song, "Assessing drivers' tailgating behavior and the effect of advisory signs in mitigating tailgating," in Proc. of the Sixth International Driving Symposium on Human Factors in Driver Assessment, Training and Vehicle Design, 2011, pp. 583-589.

[3] P. G. Michael, F. C. Leeming, and W. O. Dwyer, "Headway on urban streets: Observational data and an intervention to decrease tailgating," Transportation Research F: Traffic Psychology and Behaviour, vol. 3, no. 2, pp. 55-64, 2000. 
[4] B. Filzek and B. Breuer, "Distance behavior on motorways with regard to active safety-a comparison between Adaptive-Cruise-Control (ACC) and driver," in International Technical Conference Enhanced Safety of Vehicles, 2001.

[5] A. Vahidi and A. Eskandarian, "Research advances in intelligent collision avoidance and adaptive cruise control," IEEE Transactions on Intelligent Transportation Systems, vol. 4, pp. 143-153, 2003.

[6] T.-H. Chang and I.-S. Lai, "Analysis of characteristics of mixed traffic flow of autopilot vehicles and manual vehicles," Transportation Research Part C: Emerging Technologies, vol. 5, no. 6, pp. 333 - 348, 1997.

[7] F. Broqua, "Impact of automatic and semi-automatic vehicle longitudinal control on motorway traffic," in Proc. of the Intelligent Vehicles Symposium, 1992, pp. 144-147.

[8] S. Le Vine, A. Zolfaghari, and J. Polak, "Autonomous cars: The tension between occupant experience and intersection capacity," Transportation Research Part C, vol. 52, pp. 1 - 14, 2015.

[9] R. Mayr and O. Bauer, "Safety issues in intelligent cruise control," in IEEE/IEEJ/JSAI International Conference on Intelligent Transportation Systems, 1999, pp. 970-975.

[10] P. A. Ioannou and M. Stefanovic, "Evaluation of ACC vehicles in mixed traffic: Lane change effects and sensitivity analysis," IEEE Transactions on Intelligent Transportation Systems, vol. 6, no. 1, pp. 79-89, 2005.

[11] K. Santhanakrishnan and R. Rajamani, "On spacing policies for highway vehicle automation," IEEE Transactions on Intelligent Transportation Systems, vol. 4, no. 4, pp. 198-204, 2003.

[12] G. Marsden, M. McDonald, and M. Brackstone, "Towards an understanding of adaptive cruise control," Transportation Research Part C, vol. 9, no. 1, pp. 33 - 51, 2001.

[13] M. Eilers, C. Möbus, F. Tango, and O. Pietquin, "The learning of longitudinal human driving behavior and driver assistance strategies," Transportation Research Part F: Traffic Psychology and Behaviour, vol. 21 , pp. $295-314,2013$.

[14] Z. He, L. Zheng, and W. Guan, "A simple nonparametric car-following model driven by field data," Transportation Research Part B, vol. 80, pp. 185 - 201, 2015.

[15] K. Li and P. Ioannou, "Modeling of traffic flow of automated vehicles," IEEE Transactions on Intelligent Transportation Systems, vol. 5, no. 2, pp. 99-113, 2004.

[16] L. M. Surhone, M. T. Timpledon, and S. F. Marseken, Eds., Vienna Convention on Road Traffic. VDM Publishing, 2010.

[17] V. G. Kovvali, V. Alexiadis, and L. Zhang, "Video-based vehicle trajectory data collection," in Proc. of the Transportation Research Board 86th Annual Meeting, 2007.
[18] T. A. Dingus, S. K. Jahns, A. D. Horowitz, and R. Knipling, Human Factors in Intelligent Transportation Systems. Psychology Press, 1998, ch. Human Factors Design Issues for Crash Avoidance Systems, pp. 55-93.

[19] J. D. Lee, D. V. McGehee, T. L. Brown, and M. L. Reyes, "Collision warning timing, driver distraction, and driver response to imminent rear-end collisions in a high-fidelity driving simulator," Human Factors, vol. 44, no. 2, pp. 314-334, 2002.

[20] L. Segel, The Physics of Tire Traction. Springer, 1974, ch. Tire Traction on Dry, Uncontaminated Surfaces, pp. 65-98.

[21] C.-G. Wallman and H. Åström, "Friction measurement methods and the correlation between road friction and traffic safety," in VTI meddelande. Swedish National Road and Transport Research Institute, 2001.

[22] B. H. Wilson, "How soon to brake and how hard to brake: Unified analysis of the envelope of opportunity for rear-end collision warnings," in Proc. of the International Technical Conference on Enhanced Safety of Vehicles, 2001.

[23] F. I. A. Rizaldi and M. Althoff, "A formally verified checker of the safe distance traffic rules for autonomous vehicles," in 8th NASA Formal Methods Symposium, 2016.

[24] M. G. Lenné, T. J. Triggs, and J. R. Redman, "Time of day variations in driving performance," Accident Analysis \& Prevention, vol. 29, no. 4 pp. 431-437, Volume 29, Issue 4, July 1997, Pages 431437.

[25] G. Johansson and K. Rumar, "Drivers brake reaction times," Human Factors, vol. 13, no. 1, pp. 23-27, 1971.

[26] G. T. Taoka, "Brake reaction times of unalerted drivers," ITE journal, pp. 19-21, 1989.

[27] D. V. McGehee, E. N. Mazzae, and G. H. S. Baldwin, "Driver reaction time in crash avoidance research: Validation of a driving simulator

study on a test track," in Proc. of the Human Factors and Ergonomics Society Annual Meeting, vol. 44, no. 20. SAGE Publications, 2000, pp. 320-323.

[28] M. S. Young and N. A. Stanton, "Back to the future: Brake reaction times for manual and automated vehicles," Ergonomics, vol. 50, no. 1, pp. 46-58, 2007.

[29] M. Althoff and J. M. Dolan, "Online verification of automated road vehicles using reachability analysis," IEEE Transactions on Robotics, vol. 30, no. 4, pp. 903-918, 2014.

[30] M. Goebl, M. Althoff, M. Buss, G. Färber, F. Hecker, B. Heißing, S. Kraus, R. Nagel, F. P. León, F. Rattei, M. Russ, M. Schweitzer, M. Thuy, C. Wang, and H.-J. Wünsche, "Design and capabilities of the Munich cognitive automobile," in Proc. of the IEEE Intelligent Vehicles Symposium, 2008, pp. 1101-1107. 